\title{
Small cell carcinoma in endometrium on the base of extensive adenomyosis: differential diagnosis with immunochemistry
}

\author{
Saba Kiremitci · Korhan Kahraman • \\ Ayse Sertcelik · Firat Ortac
}

Received: 7 September 2011 / Accepted: 28 October 2011/Published online: 20 December 2011

(C) The Japan Society of Clinical Oncology 2011

\begin{abstract}
Small cell carcinomas (SCCs) are rarely seen in the endometrium and usually present as a component of a combined tumor consisting of more differentiated endometrial tumors. SCCs commonly represent the deeply invasive and aggressive component of the tumor, and differentiating SCC from counterparts has a great importance in terms of different treatment modalities and worse prognosis. Differential diagnosis includes distinct tumors indistinguishable from SCC by histologic and morphologic features, and the definite diagnosis requires immunohistochemical proof of diffuse neuroendocrine differentiation. We present a case of combined endometrial tumor composed of SCC, endometrioid adenocarcinoma, and serous carcinoma, which was initially misdiagnosed as carcinosarcoma at another institute, and discuss the differential diagnosis in terms of clinicopathologic features.
\end{abstract}

Keywords Endometrium - Neuroendocrine tumor . Small cell carcinoma

\section{Introduction}

Neuroendocrine tumors (NETs) are a well-documented heterogeneous group of neoplasms originating from NE cells, most frequently seen in the gastrointestinal tract and bronchopulmonary system [1]. The female genital tract is

S. Kiremitci · A. Sertcelik

Department of Pathology,

Ankara University School of Medicine, Ankara, Turkey

K. Kahraman $(\bowtie) \cdot$ F. Ortac

Department of Obstetrics and Gynecology,

Ankara University School of Medicine, 06100 Ankara, Turkey

e-mail: korhankahraman@hotmail.com not a common site for NETs and there have been a limited number of reports in the literature involving the most common site-the cervix, followed by the ovary [2]. However such a tumor occurring in the endometrium is very rare. Poorly differentiated (PD) NETs, i.e., small cell carcinoma (SCC) and large cell neuroendocrine carcinoma (LCNEC), are a distinct group with a high proliferation index and poor prognosis, and chemotherapy is the treatment of choice. In the endometrium, the SCC component is frequently combined with more differentiated tumors like endometrioid adenocarcinomas and serous carcinomas, and may also represent a component of malignant mixed Mullerian tumor (MMMT) [3]. A review of the literature revealed approximately 49 PD endometrial NETs (41 SCC, and 8 LCNEC), 22 of which were combined with endometrioid adenocarcinoma [4-12], 2 with adenosquamous carcinoma [6], 2 with serous carcinoma [13, 14], and 3 were a component of an MMMT [3]. But to the best of our knowledge, the concomitant existence of SCC, endometrioid adenocarcinoma, and serous carcinoma arising in the endometrium has not been previously reported in the English literature.

SCCs have a conventional histology of small-sized tumor cells with scant cytoplasm, hyperchromatic and finely granular nuclei, inconspicuous nucleoli, crushing artifacts, and high mitotic activity. However, definite diagnosis is based on the immunohistochemical (IHC) characterization of NE markers: chromogranin A, synaptophysin, CD56, and neuron-specific enolase (NSE).

We report a case of a combined endometrial tumor composed of SCC, endometrioid adenocarcinoma, and serous carcinoma on the base of extensive adenomyosis. We will discuss distinct entities in differential diagnosis which share similarities in both clinical presentation and pathologic findings. 


\section{Case presentation}

\section{Clinical presentation}

A 76-year-old, multiparous woman was referred to our gynecology department with the diagnosis of uterine MMMT from a curettage material. She had been menopausal since the age of 49 . Her medical history was significant for hypertension and coronary arterial disease. Tumor markers were normal except the slightly elevated serum CA 125 value: $53.6 \mathrm{U} / \mathrm{ml}$ (reference range less than $35 \mathrm{U} / \mathrm{ml}$ ). A chest radiograph and computed tomography scan of the thorax and upper abdomen were negative for primary or metastatic disease. A magnetic resonance imaging was not performed before the operation. An exploratory laparotomy was performed. The uterus was slightly enlarged and showed minimal irregularity on the serosal surface. Minimal serous ascites was observed. Both ovaries were atrophic. Other pelvic and abdominal structures and peritoneal surfaces were all grossly normal. A staging procedure was performed including a peritoneal washing for cytological evaluation, total abdominal hysterectomy, bilateral salpingo-oophorectomy, bilateral pelvic and para-aortic lymph node biopsy, infracolic omentectomy, and multiple peritoneal biopsies. She recovered uneventfully and was discharged on the eighth postoperative day in a stable condition. However the pathology report of the surgical specimen revealed a combined endometrial tumor with an SCC component, rather than an MMMT. She received adjuvant chemotherapy consisting of cisplatin and etoposide, and radiotherapy consisting of a total dose of $2600 \mathrm{cGY}$ (26 Gy) in 13 fractions. At the fourth postoperative month, the patient was well and disease-free in terms of uterine carcinoma.

\section{Pathology examination}

Grossly, the uterus measured $5 \times 5 \times 3.2 \mathrm{~cm}$ with a slightly irregular serosal surface. When cut open, a broadbased hemorrhagic and partially cystic polypoid lesion measuring $1 \times 0.8 \times 0.8 \mathrm{~cm}$ was identified located close to the uterine fundus. The cut surface of the polypoid lesion showed a fleshy mass deeply invasive into the thickened myometrium and serosa. The endometrium was diffusely hemorrhagic with smooth and irregular areas and measured $13 \mathrm{~mm}$ in thickness. The cut surface of the left fallopian tube showed nodular fleshy masses in the paraovarian region, whereas both ovaries were atrophic.

Microscopic examination revealed a malignant tumor composed of three distinct components (Fig. 1); the polypoid lesion corresponded to both diffusely infiltrative PD small cells and focal areas of well-formed malignant glands. The PD component constituted the large and deeply invasive portion of the tumor and was composed of densely packed small- to intermediate-sized cells with hyperchromatic nuclei, inconspicuous nucleoli, and scant cytoplasms forming solid sheets and extensive geographic tumor necrosis. Nuclear molding, crushing artifact, and high mitotic rate were evident. The endometrioid adenocarcinoma component was composed of glandular and villoglandular foci with no evidence of squamous metaplasia. The third and minor component of the tumor was composed of cells with a high degree of cytological atypia and micropapillary appearance consistent with serous carcinoma (Fig. 1b). A striking finding was that the uterine wall revealed extensive adenomyosis comprising tubular and cystic atrophic glands, and tumor components were intimately nested within the adenomyotic foci some of which exhibited malignant transformation of atrophic epithelium to malignant epithelium in the same gland (Fig. 2). Lymphovascular invasion was prominent. The right ovary and left tube were infiltrated by the PD component of the tumor. There were only microscopic tumor foci in the former, whereas the wall of the tube was diffusely infiltrated reaching up to the subepithelial region. The tumor possessed no sarcomatous or heterologous component upon thorough examination. Lymph node involvement was confined to only one (1/6) left pelvic lymph node and the metastasis was in serous carcinoma phenotype. The omentum was free of tumor. The cytology of the peritoneal fluid was negative for malignant cells.

A wide IHC panel was applied to identify the nature of the tumor components, particularly the PD one. Antibodies and the staining properties of the tumor components are summarized in Table 1. IHC results demonstrated a different pattern of protein expression in the two components (Fig. 3); the PD component was diffusely positive with synaptophysin, CD56, and vimentin, and focally with chromogranin A, whereas the adenocarcinoma component was positive with only PANCK and vimentin. CD10 was expressed only in endometrial stromal cells in residual adenomyosis foci. Ttf-1, caldesmon, SMA, and desmin were all negative. Hematoxylin and eosin (H\&E) slides of the previous endometrial curettage obtained from the previous institute showed intermingled serous carcinoma and undifferentiated areas with no sarcomatous foci. We realized that the undifferentiated component was misinterpreted as sarcomatous differentiation and thereby a diagnosis of MMMT was made without the assistance of IHC in the previous investigation.

Strong and consistent expression of NE markers (chromogranin A, synaptophysin, and CD56) and the cytological features proved the PD component to be a SCC, whereas other components were consistent with endometrioid adenocarcinoma and serous carcinoma on the basis of their characteristic histological appearance. A final diagnosis 

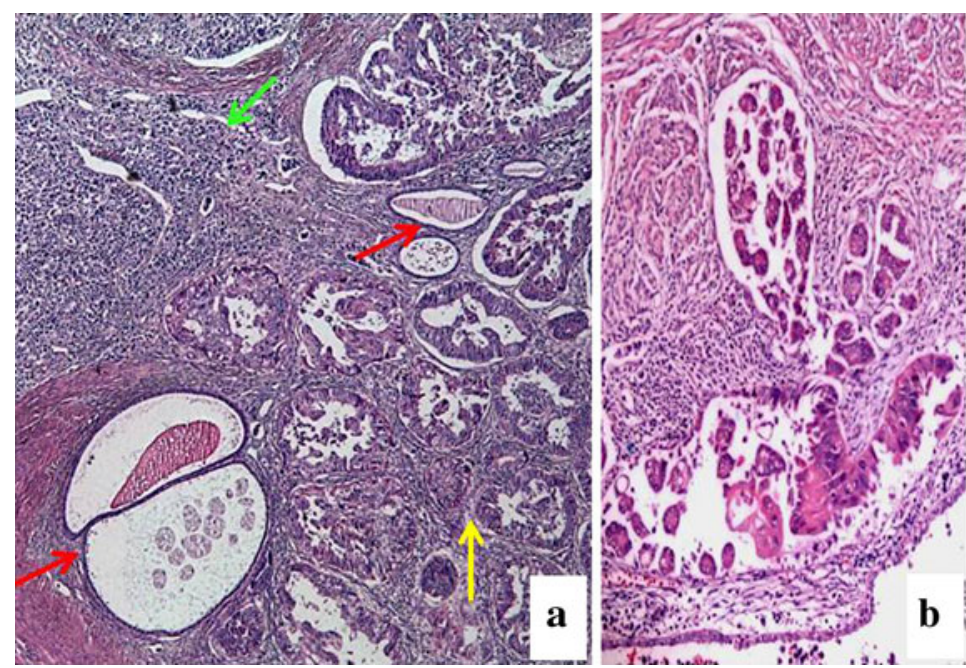
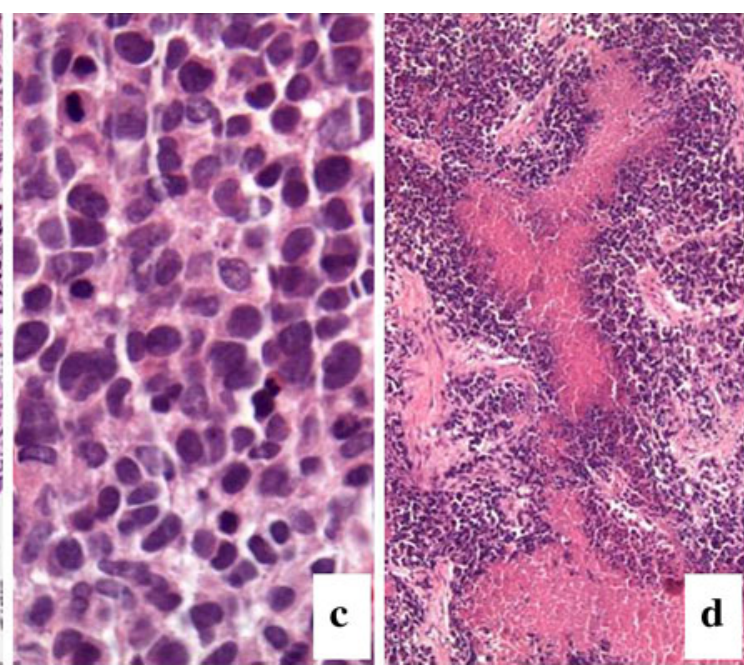

Fig. 1 Histologically tumor showed three different components: a PD component (green arrow) and well-differentiated endometrioid adenocarcinoma component (yellow arrow) were admixed and grew up on the basis of adenomyosis (red arrows). b The third component was viewed in limited areas with small micropapillary morphology and significant cytologic atypia. c PD component was highly cellular composed of small cells with high nucleocytoplasmic ratio and high mitotic activity, and showed extensive geographic tumor necrosis (d). $\mathrm{H} \& \mathrm{E} \times 40, \times 100, \times 400, \times 100$, respectively

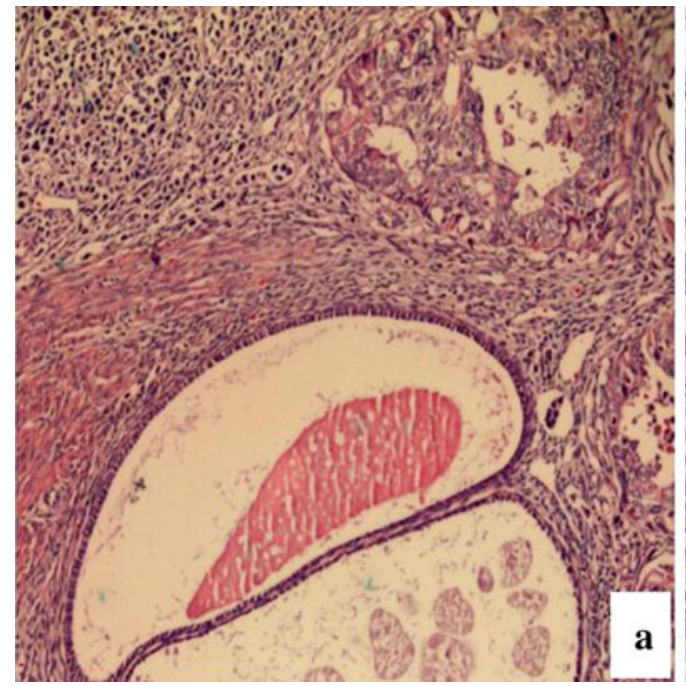

Fig. 2 PD component diffusely infiltrated the uterine wall, and the remaining endometrial glands, both in the atrophic endometrium and adenomyosis foci, revealed various histomorphology including

compatible with a combined tumor of SCC, endometrioid adenocarcinoma, and serous carcinoma of 2009 FIGO (Federation Internationale de Gynecologie et d'Obstetrique) stage IIIC was achieved.

\section{Discussion}

Surgical material of the present case revealed an aggressive tumor with three distinct components: SCC, endometrioid adenocarcinoma, and serous carcinoma. The predominant atrophic cystic (a), tubular-proliferative (b), and atypical (c) glands (transition to malignant epithelium in an atrophic gland; arrow), intermingled with the three components of tumor. $\mathrm{H} \& \mathrm{E} \times 200$

component, SCC, fulfilled the three diagnostic criteria proposed by Van Hoeven et al. [5]: no evidence of primary origin from the endometrium, characteristic histology with small- to intermediate-sized tumor cells growing in a dense, sheet-like fashion, and immune reactivity for the NE markers synaptophysin, CD56, and chromogranin A. As in our case, focal chromogranin A positivity was reported previously [10, 12]. SCC was the aggressive component as was evident by its full-thickness myometrial invasion and diffuse endometrial stromal invasion leaving residual endometrial glands with or without atypia. Metastatic 
Table 1 Immunohistochemical characteristics of tumor components

\begin{tabular}{|c|c|c|c|c|c|c|}
\hline \multirow[t]{2}{*}{ Primary antibody } & \multirow[t]{2}{*}{ Clone } & \multirow[t]{2}{*}{ Dilution } & \multirow[t]{2}{*}{ Source } & \multirow[t]{2}{*}{ Cellular localization } & \multicolumn{2}{|l|}{ Tumor components } \\
\hline & & & & & PD & Adenocarcinoma \\
\hline PANCK & SD3 + LP34 & $1 / 400$ & Neomarkers & Cytoplasmic & Negative & Diffuse strong staining \\
\hline Vimentin & V9 & $1 / 200$ & Neomarkers & Cytoplasmic & Diffuse strong staining & Diffuse strong staining \\
\hline Synaptophysin & Polyclonal & $1 / 50$ & Invitrogen & Cytoplasmic, membranous & Diffuse strong staining & Negative \\
\hline Chromogranin A & Cocktail & $1 / 1000$ & Neomarkers & Cytoplasmic, membranous & Focal moderate staining & Negative \\
\hline CD56 & $\mathrm{S} 6 \mathrm{CO} 4$ & $1 / 100$ & Neomarkers & Cytoplasmic, membranous & Diffuse strong staining & Negative \\
\hline TTF-1 & $8 \mathrm{G} 763 / 1$ & $1 / 250$ & Cell Marque & Nuclear & Negative & Negative \\
\hline Desmin & DE-R-11 & $1 / 150$ & Novocastra & Cytoplasmic & Negative & Negative \\
\hline Caldesmon & H-Cald & $1 / 400$ & Neomarkers & Cytoplasmic & Negative & Negative \\
\hline SMA & $1 \mathrm{~A} 4$ & $1 / 500$ & Dako & Cytoplasmic & Negative & Negative \\
\hline
\end{tabular}
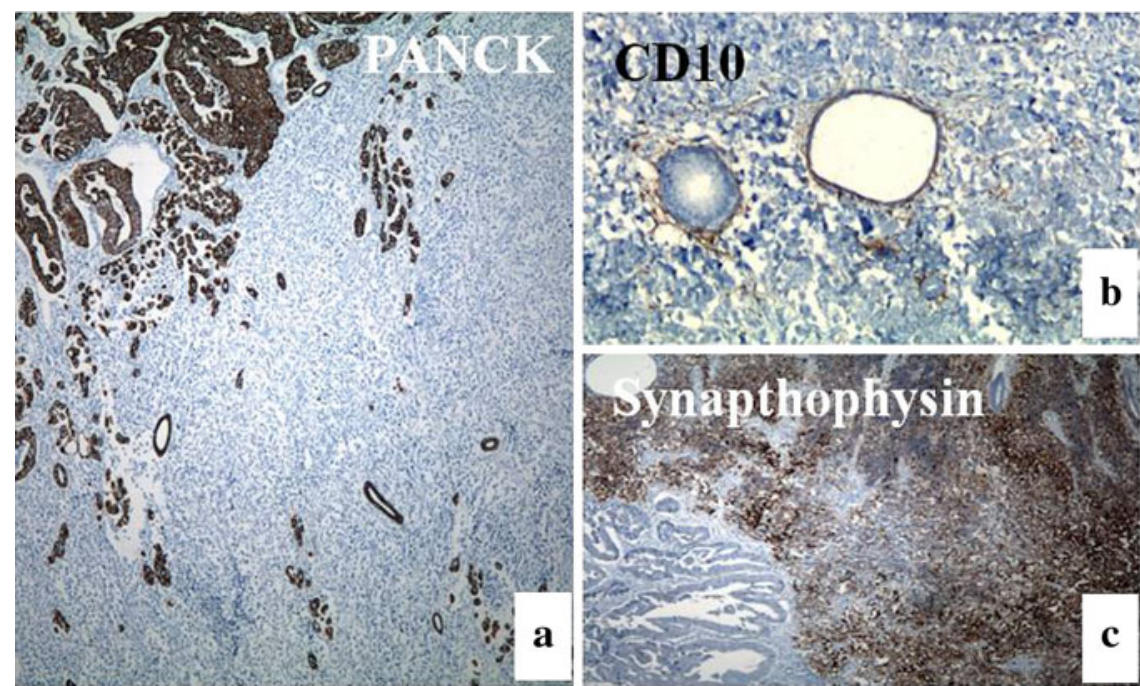

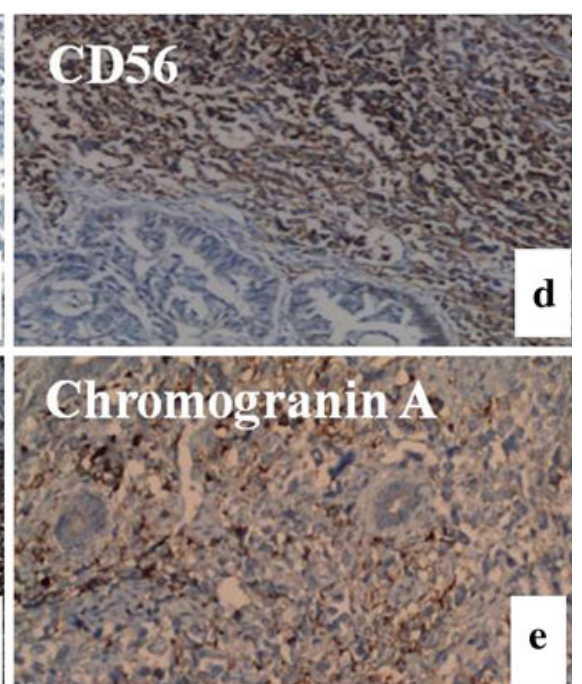

Fig. 3 PD component exhibited a discriminating immunoreactivity; PANCK highlighted both the adenocarcinoma component and nonneoplastic endometrial glands remaining in the adenomyosis foci infiltrated diffusely by PD component (a). CD10 showed up the endometrial stromal cells surrounding the non-neoplastic endometrial glands as evidence of adenomyosis (b). Synaptophysin (c), CD56 (d) (diffusely, with strong intensity), and chromogranin A (e) (focally, with moderate intensity) were expressed in the PD component, whereas the adenocarcinoma components were entirely negative for these markers. Immuno peroxidase; $\times 40, \times 100, \times 40, \times 100, \times 200$, respectively

other in terms of clinical presentation and gross and microscopic appearances. Diverse endometrial tumors, particularly MMMT and endometrial stromal sarcoma, commonly present with bulky, friable polypoid masses that involve the uterine wall deeply, fill the uterine cavity, and protrude through the external os, as a common finding shared with SCC. MMMT may occur in elderly postmenopausal women with extrauterine disease at initial presentation, like SCC.

As an initial approach, the possibility of metastatic carcinoma of the endometrium should be excluded by using detailed clinical information and radiology findings. SCCs exhibit the same histology independently from the primary site. The lung is the most common site and it is not 
surprising for a primary lung SCC to accompany an adenocarcinoma. However, IHC evidence of Ttf1 expression would reliably indicate pulmonary origin [15]. Endometrial SCCs also differ from pulmonary SCCs by diffuse expression of vimentin $[8,16]$, with the exception of a unique report exhibiting vimentin negativity in a primary endometrial SCC [12]. Cytokeratin expression is not differentiating, and is variable in SCCs. Endometrial carcinomas should also be investigated for an ovarian primary disease.

In the present case, the initial pathologic diagnosis in endometrial curettage was interpreted as MMMT, at an outside center. The SCC component of a combined tumor may be misdiagnosed as undifferentiated sarcoma, leading a diagnostic dilemma with MMMT. MMMTs have a biphasic appearance both with carcinomatous and sarcomalike elements. The former may exhibit any type of endometrial carcinoma commonly including endometrioid and serous carcinoma, whereas the latter may reveal homologous (fibrosarcoma or leiomyosarcoma) or heterologous (chondrosarcoma, osteosarcoma, or rhabdomyosarcoma) elements. Detailed IHC examination with desmin, SMA, myoglobin, and S100 antibodies would differentiate the sarcomatous component of MMMTs, in general. Differentiation of SCC from sarcoma definitely requires IHC evidence of NE markers. NE differentiation in MMMTs is particularly seen as focal expression which is distinct from the diffuse expression observed in SCCs [17].

Mullerian adenosarcoma, which defines the benign glandular component admixed with malignant mesenchymal component, should also be kept in mind in the differential diagnosis. In the present case, the SCC component invaded the endometrium leaving residue non-neoplastic and atrophic glands, and even in adenomyosis areas, leading to a misdiagnosis of an adenosarcoma. A malignant epithelial component of a combined tumor may be overlooked especially in curettage materials and also inadequate sampling of the tumor may lead to a misdiagnosis of MMMT or a combined tumor as adenosarcoma.

Endometrial undifferentiated carcinoma, an aggressive tumor commonly presenting with an advanced stage, is a controversial entity that fails to show evidence of glandular or squamous differentiation according to the World Health Organization definition [18]. It is commonly associated with an endometrioid adenocarcinoma and, thus, may be misdiagnosed as a high-grade endometrioid adenocarcinoma component. However, diagnosis of high-grade endometrioid adenocarcinoma requires the definite presence of glands which is not an expected finding for endometrial undifferentiated carcinoma [19]. The NE component of a combined tumor, particularly LCNEC, may be misinterpreted as an endometrial undifferentiated carcinoma because of its similar cytological features.
Nevertheless, IHC evidence of diffuse NE differentiation would distinguish SCC from the latter which may also exhibit NE differentiation, but in a limited number of tumor cells, approximately $10 \%$ [20].

Endometrial stromal sarcomas are indolent tumors composed of uniform, small, oval cells with scanty cytoplasm and may metastasize even many years after initial diagnosis. Their differentiation from endometrial combined tumors is relatively straightforward on the basis of absence of significant cytological atypia and resemblance to normal proliferative phase endometrium with the characteristic feature of regularly distributed spiral arterioles. Furthermore, endometrial stromal sarcomas commonly occur in premenopausal women in contrast to the aforementioned tumors. CD10 has been considered as a specific marker for both neoplastic and non-neoplastic endometrial stromal cells [21], and probably distinguishes endometrial stromal neoplasms from others. However, there has been a report stressing CD10 positivity both in MMMT and Mullerian adenosarcoma, and indicating that CD10 is a characteristic of Mullerian system-derived neoplastic mesenchymal cells [22].

Lymphoma and ES/PNET are practically distinguished by IHC analysis of LCA antibody for the former, and CD99 for the latter.

In conclusion, it has long been known that diverse endometrial tumors with different prognosis and therapeutic modalities may reveal similar histopathologic findings. Although characteristic histological features may usually lead to a correct diagnosis, the NE component may be misdiagnosed as undifferentiated carcinoma or sarcoma, particularly in the absence of IHC analysis. Therefore, it is advisable to carry out IHC in the histopathology examination of the combined tumors to avoid misinterpretations.

Conflict of interest The authors declare that they have no conflict of interest.

\section{References}

1. Barakat MT, Meeran K, Bloom SR (2004) Neuroendocrine tumors. Endocr Relat Cancer 11:1-18

2. Eichhorn JH, Young RH (2001) Neuroendocrine tumors of the genital tract. Am J Clin Pathol 115(Suppl 1):94-112

3. Manivel C, Wick MR, Sibley RK (1985) Neuroendocrine differentiation in mullerian neoplasms. An immunohistochemical study of a "pure" endometrial small-cell carcinoma and a mixed mullerian tumor containing small-cell carcinoma. Am J Clin Pathol 85:438-443

4. Huntsman DG, Clement PB, Gilks CB et al (1994) Small cell carcinoma of the endometrium. A clinicopathological study of sixteen cases. Am J Surg Pathol 18:364-375

5. Van Hoeven KH, Hudock JA, Woodruff JM et al (1995) Small cell neuroendocrine carcinoma of the endometrium. Int J Gynecol Pathol 14:21-29 
6. Katahira A, Akahira J, Nikura H et al (2004) Small cell carcinoma of the endometrium: report of three cases and literature review. Int J Gynecol Cancer 14:1018-1023

7. Rajab KE, Sandhu AK, Malik A et al (2005) Small cell neuroendocrine carcinoma of the endometrium, a rare aggressive tumor. Saudi Med J 26:1130-1132

8. Melgoza F, Brewster WR, Wilczynski S et al (2006) p16-Positive small cell neuroendocrine carcinoma of the endometrium. Int $\mathrm{J}$ Gynecol Pathol 25:252-256

9. Mulvany NJ, Allen DG (2007) Combined large cell neuroendocrine and endometrioid carcinoma of the endometrium. Int $\mathrm{J}$ Gynecol Pathol 27:49-57

10. Bige O, Saatli B, Secil M et al (2008) Small cell neuroendocrine carcinoma of the endometrium and laparoscopic staging: a clinicopathologic study of a case and a brief review of the literature. Int J Gynecol Pathol 18:838-843

11. Albores-Saavedra J, Martinez-Benitez B, Luevano E (2008) Small cell carcinomas and large cell neuroendocrine carcinomas of the endometrium and cervix: polypoid tumors and those arising in polyps may have a favorable prognosis. Int J Gynecol Pathol 27:333-339

12. Hwang JH, Lee JK, Lee NW et al (2010) Primary small cell carcinoma of the endometrium: report of a case with immunohistochemical studies. J Reprod Med 55:81-86

13. Shaco-Levy R, Manor E, Piura B et al (2004) An unusual composite endometrial tumor combining papillary serous carcinoma and small cell carcinoma. Am J Surg Pathol 28:1103-1106

14. Posligua L, Malpica A, Liu J et al (2008) Combined large cell neuroendocrine carcinoma and papillary serous carcinoma of the endometrium with pagetoid spread. Arch Pathol Lab Med 132:1821-1824

15. Travis WB, Nicholson S, Hirsch FR et al (2004) Small cell carcinoma. In: Travis W, Brambilla E, Muller-Hermelink HK et al (eds) Pathology and genetics. Tumors of the lung, pleura, thymus and heart. World Health Organization classification of tumors. IARC, Lyon, pp 31-34

16. Upton MP, Hirohashi S, Tome Y et al (1986) Expression of vimentin in surgically resected adenocarcinomas and large cell carcinomas of lung. Am J Surg Pathol 10:560-567

17. George E, Manivel JC, Dehner LP et al (1991) Malignant mixed mullerian tumors: an immunohistochemical study of 47 cases, with histogenetic considerations and clinical correlation. Hum Pathol 22:215-223

18. Silverberg SG, Kurman RJ, Nogales F et al (2003) Epithelial tumors and related lesions. In: Tavassoli FA, Devilee P (eds) Tumors of the breast and female genital organs: World Health Organization classification of tumors. IARC, Lyon, p 227

19. Altrabulsi B, Malpica A, Deavers MT et al (2005) Undifferentiated carcinoma of the endometrium. Am J Surg Pathol 2:1316-1321

20. Silva EG, Deavers MT, Malpica A (2007) Undifferentiated carcinoma of the endometrium: a review. Pathology 39:134-138

21. Toki T, Shimizu M, Takagi Y et al (2002) CD10 is a marker for normal and neoplastic endometrial stromal cells. Int J Gynecol Pathol 21:41-47

22. Mikami Y, Hata S, Kiyokawa T et al (2002) Expression of CD10 in malignant mullerian mixed tumors and adenosarcomas: an immunohistochemical study. Mod Pathol 15:923-930 